\section{Experimental and numerical approach for the investigation of interface pressure applied by compression bandages}

\section{Fanette Chassagne, ${ }^{1-4}$ Pierre Badel, ${ }^{1-3}$ Reynald Convert, ${ }^{4}$ Pascal Giraux, ${ }^{5}$ Jérôme Molimard ${ }^{1-3}$}

${ }^{1}$ Ecole Nationale Supérieure des Mines de Saint-Etienne, CIS-EMSE, SAINBIOSE, F-42023 Saint Etienne, France; ${ }^{2}$ Inserm, U1059, Saint-Etienne, F-42000, France; ${ }^{3}$ Université de Lyon, SAINBIOSE, F-42000 Saint Etienne, France; ${ }^{4}$ Thuasne, BP243, 92307 LevalloisPerret, France; 5 Department of Physical Medicine and Rehabilitation, Faculty of Medicine, University Jean Monnet, Saint-Etienne, France

\section{Introduction}

Compression bandages are a common treatment for some venous or lymphatic pathologies, whose efficacy is admitted. ${ }^{1}$ The bandage applies a pressure on the external surface of the leg which is then transmitted to the internal tissues. This interface pressure depends on several parameters such as: i) the bandage components, the application technique, their mechanical properties and stretch; ii) the patient's morphology and soft tissue mechanical properties; iii) other parameters like friction for example.

Various bandages are proposed by manufacturers and their study requires reliable methods to predict interface pressure applied by these bandages. For now, the only method for interface pressure computation is Laplace's Law:

$P=n T / r, \quad T>0, \quad r>0$

with $P$ the pressure $\left(N * m m^{-2}\right), n$ the number of layers of the bandage, $T$ the bandage tension (ratio between the force needed to stretch the bandage and the bandage width) $\left(N * \mathrm{~mm}^{-1}\right)$ and $r$ the local radius of curvature $(\mathrm{mm})$. However, this equation is questioned in the community. 2,3 This work aims to propose an original point of view on this problem.

We propose an experimental and numerical approach for the investigation of interface pressure applied by compression bandages.

\section{Materials and Methods}

\section{Interface pressure measurements}

Pressure measurements were performed on 30 healthy subjects (15 males - 15 females) in order to evaluate the impact of bandage mechanical properties, application technique and subject's morphology on the interface pressure applied by elastic compression bandages. These measurements were performed on the medial part of the leg at the heights of measurement point B1 and $\mathrm{C}$ thanks to pressure sensors Picopress ${ }^{\circledR}$ (Microlab Elettronica, Nicolò PD, Italy). Two elastic bandages were used in this study: the Biflex ${ }^{\circledR} 16$ (B16) and Biflex ${ }^{\circledR} 17$ (B17) (manufactured by Thuasne), which were applied on the leg in a spiral pattern with 2 or 3 layers (i.e. $50 \%$ and $66 \%$ overlap) and with a 1.3 stretch (following manufacturer's recommendations). These bandages have a similar structure but differ in their elastic moduli (which links the bandage tension to its stretch), respectively equal to 0.232 and $0.444 \mathrm{~N} / \mathrm{mm}$ for the B16 and the B17.4

\section{Numerical simulation}

Whitin the aim to predict interface pressure applied by compression bandages, a patient-specific numerical simulation was designed for 5 female subjects.

For this, their leg geometries were obtained with a 3D optical scanner (Artec $3 \mathrm{D}^{\circledR}$ scanner) and the bandage geometry was built in Matlab $^{\circledR}$, in order to fulfil the 1.3 stretch requirement. Bandage model was inflated then relaxed around the sub-
Correspondence: Fanette Chassagne, Ecole Nationale Supérieure des Mines de SaintEtienne, CIS-EMSE, SAINBIOSE, F-42023 Saint Etienne, France.

E-mail: fanette.chassagne@emse.fr

This work is licensed under a Creative Commons Attribution 4.0 License (by-nc 4.0).

CCopyright F. Chassagne et al., 2017

Licensee PAGEPress, Italy

Veins and Lymphatics 2017; 6:6626

doi:10.4081/vl.2017.6626

ject's leg model.

The simulation was run for four bandages: B16 and B17 applied in a spiral pattern with 2 and 3 layers (Figure 1).

Interface pressure given by the numerical simulations was then confronted to the experimental values and to those computed with Laplace's law.

\section{Results}

\section{Interface pressure measurements}

The interface pressure measurements showed a very strong correlation between pressure and bandage tension $(\mathrm{P}<0.00001)$ and between pressure and bandage overlapping $(\mathrm{P}<0.00001)$. Moreover interface pressure tends to decrease when leg circumference increases. However, these results highlighted the fact that interface pressure is not directly proportional to bandage elastic moduli and so not proportional to bandage
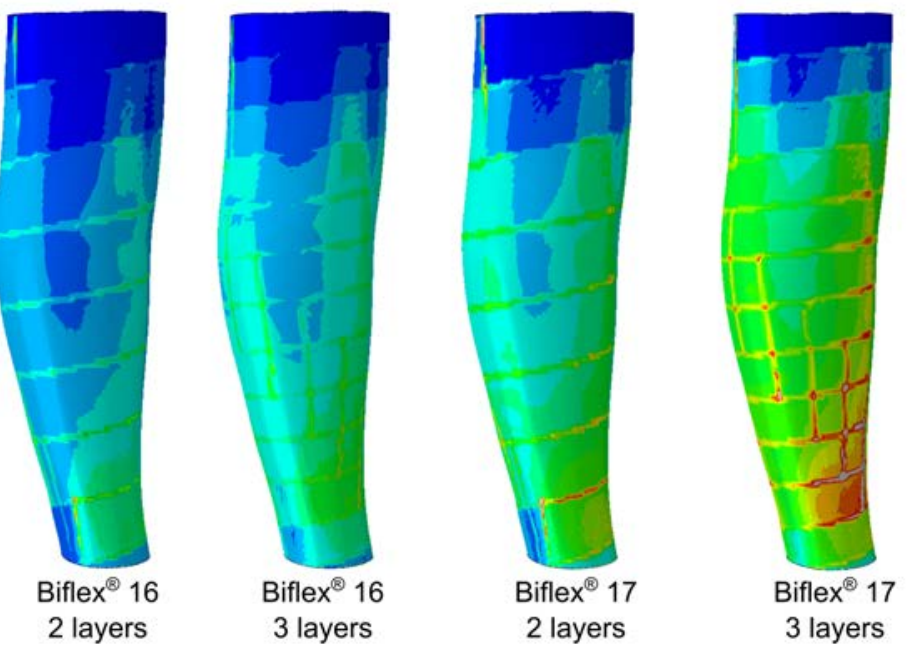

Pressure [mmHg]

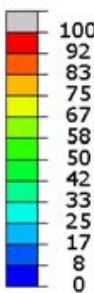

Figure 1. Pressure distribution over a leg for 4 different bandages. 
tension nor to the leg circumference and consequently to a global estimate of the radius of curvature. These results question the use of Laplace's Law for interface pressure computation and support the need to develop new tools for interface pressure computation.

\section{Numerical simulation}

Numerical model differs significantly from Laplace Law, with differences up to $19.6 \%$. The main difference between the two models being the mechanical behaviour of the leg, it is proposed that the leg shape variation due to compression plays an important role in pressure generation through changes in curvature radii. Nevertheless, significant discrepancy between numerical model and experiments are reported. It is likely that the model relevance would be increased by improving the simulation of bandage application tech- nique. Besides, the presence of the pressure sensors might locally change the pressure by modifying the local curvature.

\section{Discussion and Conclusions}

Laplace's law does not take into account the mechanical effects involved in bandage application. More specifically, it has been shown that changes in the leg geometry influence the applied pressure.

This study demonstrated that, using an appropriate surrogate model, such a simulation could be an efficient tool for interface pressure prediction.

Future works will be focused on improving the simulation and investigating possible errors due to the pressure sensors.

\section{References}

1. Amsler F, Willenberg T, Blättler W. In search of optimal compression therapy for venous leg ulcers: a meta-analysis of studies comparing divers bandages with specifically designed stockings. J Vasc Surg 2009;50:668-74.

2. Schuren J, Mohr K. The efficacy of Laplace's equation in calculating bandage pressure in venous leg ulcers. Wounds 2008;4:38-47.

3. Thomas S. The production and measurement of sub-bandage pressure: Laplace's Law revisited. J Wound Care 2014;23:234-6, 238-41, 244.

4. Chassagne F, Martin F, Badel P, et al. Experimental investigation of pressure applied on the lower leg by elastic compression bandage. Ann Biomed Eng 2015;43:2967-77. 